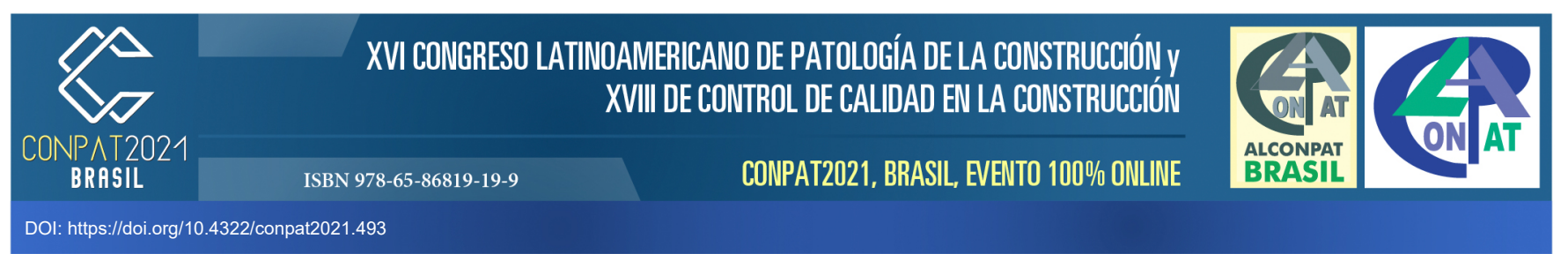

\title{
ESTUDO DA AÇÃO DE AGENTES DE DEGRADAÇÃO DE FACHADAS ASSOCIADOS À TEMPERATURA E A CHUVA DIRIGIDA EM DIFERENTES ZONAS BIOCLIMÁTICAS BRASILEIRAS.
}

\author{
A.L. $\operatorname{Ramos}^{1 *}$, E. Bauer ${ }^{2}$ \\ *Autor de Contato: analin.ramos@email.com \\ ${ }^{1}$ Aluna de mestrado PECC, Universidade de Brasília, Brasília, Brasil \\ ${ }^{2}$ Professor Doutor do PECC,Universidade de Brasília, Brasília, Brasil
}

\section{RESUMO}

Compreender a ação do intemperismo é essencial para entender a degradação das fachadas. Logo, objetiva-se estudar as condições de exposição a esses agentes de degradação em edifícios localizados em diferentes zonas bioclimáticas brasileiras. Para tanto, selecionou-se oito cidades representativas de cada Zona: Curitiba, Santa Maria, Florianópolis, Brasília, Niterói, Goiânia, Picos e Belém . Definiu-se um edifício modelo para simulação higrotérmica para avaliação da radiação total, amplitude térmica, choque térmico, índice de intensidade da temperatura e chuva dirigida. Como resultado, são identificadas as zonas de condições críticas, fornecendo rankings de exposição. Para os agentes de temperatura e chuva dirigida, as zonas mais expostas são Z6 e Z8, respectivamente. Por fim, as zonas de exposição mais amenas são Z8 para agente de temperatura e Z5 para chuva dirigida.

Palavras chave: Degradação; Fachadas; Revestimento cerâmico; Zonas bioclimáticas; temperatura.

\section{RESUMEN}


Conocer la acción de la meteorización es fundamental para comprender la degradación de las fachadas. Por tanto, el objetivo es estudiar las condiciones de exposición a estos agentes degradantes en edificios ubicados en diferentes zonas bioclimáticas brasileñas. Para ello, se seleccionaron ocho ciudades: Curitiba, Santa María, Florianópolis, Brasília, Niterói, Goiânia, Picos y Belém.Se definió un modelo de construcción de simulación higrotérmica para evaluar radiación total, amplitud térmica, choque térmico, índice de intensidad de temperatura y lluvia dirigida. Como resultado, se identifican zonas de condiciones críticas, proporcionando clasificaciones de exposición. Para agentes de temperatura y lluvia dirigida, las zonas más expuestas son Z6 y Z8, respectivamente. Finalmente, las zonas de exposición más suaves son Z8 para agente de temperatura y Z5 para lluvia dirigida.

Palabras llave: Degradación; Fachadas; Revestimiento ceramico; Zonas bioclimáticas; temperatura..

\section{ABSTRACT}

Knowing the action of weathering is essential to understand the facades degradation. Therefore, the aim is to study the conditions of exposure to these degradation agents in buildings located in different Brazilian bioclimatic zones. For this purpose, eight cities were selected: Curitiba, Santa Maria, Florianópolis, Brasília, Niterói, Goiânia, Picos and Belém. A model building was defined for hygrothermal simulation to assess total radiation, temperature range, thermal shock, intensity index temperature and driving rain. As a result, zones of critical conditions are identified, providing exposure rankings. For temperature agents and directed rain, the most exposed zones are Z6 and Z8, respectively. Finally, the mildest exposure zones are Z8 for temperature agent and $\mathrm{Z} 5$ for driving rain.

Keywords: Degradation; Facades; Ceramic coating; Bioclimatic zones; temperature.

\section{INTRODUÇÃO}

A fachada se configura como um elemento construtivo de proteção da edificação ao ambiente externo tendo como consequência a sua maior exposição aos fatores de intemperismos como radiação solar, chuva dirigida e temperatura. Tal exposição possibilita um processo de degradação complexo que envolve agentes de diferentes naturezas, que podem atuar em sinergia, afetando o desempenho e vida útil dos componentes e materiais por meio de diferentes mecanismos de degradação.

O uso de revestimentos cerâmicos em fachadas é comum e está associado a um processo de degradação específico caracterizado pela fissuração, falta de adesão cerâmica-substrato e pela expansão de substratos devido à umidade ou variações térmicas (Bezerra et al., 2018). Nesse caso, as camadas que compões o sistema de revestimento apresentam propriedades distintas (coeficientes de dilatação térmica, módulo de elasticidade, condutividade térmica, etc) e a ação agentes associados à temperatura apresentam alta relevância (Gaspar e Brito, 2011).

A variação da temperatura superficial do revestimento pode causar alterações físicas no sistema de vedação de fachada. Esse mecanismo de degradação se dá a partir do efeito de dilatação e contração pelas variações e incrementos não homogêneos de temperatura, o que causa esforços de cisalhamento gerando uma tendência de separação na interface do sistema (Saraiva, 1998). Como resultado tem-se descolamentos e fissuras no revestimento cerâmico, o que pode ser intensificado pela fadiga causada o mecanismo cíclico da temperatura (Barbosa, 2013). 
Desse modo, foram calculados como valores representativos da amplitude térmica e para indicar uma maior degradação o Índice de intensidade da temperatura (Iit), radiação solar e choques térmicos. O Iit constitui uma análise da amplitude térmica obtendo-se uma medida ponderada em relação à frequência (Nascimento, 2016) e o choque térmico são eventos pontuais caracterizados por uma alta diferença de temperatura superficial em um curto intervalo de tempo (Zanoni, 2015).

Além disso, considera-se como agente de grande relevância a ação da chuva dirigida sob as fachadas. Esta se origina a partir da associação da chuva com o vento, e esta é uma das principais fontes de água nas fachadas dos edifícios. Sem a ação do vento a chuva cairia no sentido vertical e molharia de forma pouco significativa as paredes (Zanoni, 2015). Por causa da ação do vento, a chuva atinge principalmente as partes superior e lateral dos edifícios. Desse modo, é importante considerar esse fator nas simulações higrotérmicas para se compreender o processo de degradação, principalmente em materiais porosos que permitem o transporte de água por meio de capilaridade.

A degradação de sistemas de revestimentos cerâmicos é caracterizada principalmente pelo descolamento cerâmico, ainda que se considerem mudanças no processo de degradação para diferentes localizações geográficas dos edifícios (Souza, 2019). Por isso é necessário o estudo das condições de exposição ás ações de agentes climáticos de forma a compreender as diferentes condições que desencadeiam o processo de degradação em diferentes regiões.

O Brasil, como um país continental, apresenta variações climáticas ao longo de seu território. Por esta razão a ABNT NBR 15220-3 (2005) estabelece o zoneamento bioclimático brasileiro, classificando 330 cidades a partir de seu clima em oito zonas bioclimáticas. Estas são definidas como regiões geográficas homogêneas quanto aos elementos climáticos que interferem nas relações entre ambiente construído e conforto humano. Nesse sentido, as edificações e seus elementos localizados em zonas diferentes estão condicionados a diferentes condições de exposição aos agentes climáticos que ocasionam o processo de degradação.

Dentro desse contexto, o referente trabalho tem como objetivo investigar a ação dos agentes de degradação associados à temperatura e a chuva dirigida nas fachadas de edifícios localizados em diferentes zonas bioclimáticas brasileiras. Para tanto, se emprega a simulação higrotérmica pelo software WUFI Pro 6.5, de radiação solar, amplitude térmica, choque térmico, índice de intensidade da temperatura e chuva dirigida das fachadas Norte de um edifício modelo submetido às diferentes condições climáticas de cada zona.

\section{MÉTODOS}

\subsection{Detalhes do edifício modelo}

Determinou-se um edifício modelo de 20 metros de altura com sistema de revestimento cerâmico de absortância 63,5\% com base em Dornelles (2007) correspondente a cor vermelho escuro, as quais foram escolhidas para ressaltar os efeitos da temperatura possibilitando comparações. Aplica-se este modelo a cidades localizadas nas oito zonas bioclimáticas brasileiras estabelecidas na ABNT NBR 15220-3 (2005), por meio de simulação no software WUFI Pro 6.5. A Tabela 1 mostra as cidades escolhidas e a zona bioclimática correspondente.

Tabela1. Cidade, Estado e Zonas bioclimáticas. 


\begin{tabular}{|c|c|c|c|c|c|c|c|}
\hline $\mathbf{Z 1}$ & $\mathbf{Z 2}$ & $\mathbf{Z 3}$ & $\mathbf{Z 4}$ & $\mathbf{Z 5}$ & $\mathbf{Z 6}$ & $\mathbf{Z 7}$ & $\mathbf{z 8}$ \\
\hline $\begin{array}{c}\text { Curitiba- } \\
\text { PR }\end{array}$ & $\begin{array}{c}\text { Santa Maria- } \\
\text { RS }\end{array}$ & $\begin{array}{c}\text { Florianópolis- } \\
\text { SC }\end{array}$ & $\begin{array}{c}\text { Brasília- } \\
\text { DF }\end{array}$ & $\begin{array}{c}\text { Niterói- } \\
\text { RJ }\end{array}$ & $\begin{array}{c}\text { Goiânia- } \\
\text { GO }\end{array}$ & $\begin{array}{c}\text { Picos- } \\
\text { PI }\end{array}$ & $\begin{array}{c}\text { Belém- } \\
\text { PA }\end{array}$ \\
\hline
\end{tabular}

\subsection{Simulação}

A simulação higrotérmica foi realizada no programa WUFI Pro 6.5 no sentido de avaliar a ação dos agentes climáticos nas fachadas em cada cidade. O programa fornece uma interface simples que permite a entrada de dados, os quais são: período de simulação, configuração, orientação e inclinação do sistema construtivo, coeficientes de chuvas incidentes e de transferência à superfície e condições iniciais de temperatura e umidade relativa e condições climáticas interiores e exteriores (Freitas, 2011). A partir disso, foram obtidos como dados horários de saída os valores de radiação total incidentes nas fachadas, de temperatura superficial e de precipitação.

O período de simulação foi de um ano de 01/01/2019 a 01/01/2020. Já para a definição das condições climáticas exteriores foram utilizados os dados arquivos Typical Meteorological Year (TMY) da base EPW/ANTAC (RORIZ, 2012) referente a cada cidade. A tabela 2 contêm os valores adotados dos coeficientes de transferência à superfície.

Tabela 2. Coeficientes de transferência à superfície externa adotados na simulação.

\begin{tabular}{|c|c|c|}
\hline Coeficiente & Unidade & Valor \\
\hline Resistência Térmica (lado esquerdo) & $\mathrm{m}^{2} \mathrm{~K} / \mathrm{W}$ & 0,058 \\
\hline Valor de sd* & $\mathrm{M}$ & - \\
\hline Absorção (Radiação de onda curta) & - & 0,635 \\
\hline Refletividade do solo & - & 0,2 \\
\hline Fator de redução de chuva incidente & - & 0,7 \\
\hline $\begin{array}{l}* \text { O valor de Sd é a espessura de camada de ar de difusão equivalente. Não foi } \\
\text { espessificado, pois não são consideradas camadas muito finas como pinturas. }\end{array}$ \\
\hline
\end{tabular}

No estudo, optou-se por analisar apenas a orientação Norte de cada edifício, uma vez que esta se apresenta como a mais crítica para incidência de radiação solar no caso das cidades estudadas, as quais estão situadas abaixo da linha do equador. O sistema construtivo simulado é composto por: revestimento cerâmico, argamassa de emboço, bloco cerâmico e argamassa interna e o ponto de monitoramento principal na superfície do revestimento. A Figura 1 mostra o sistema lançado no programa. 


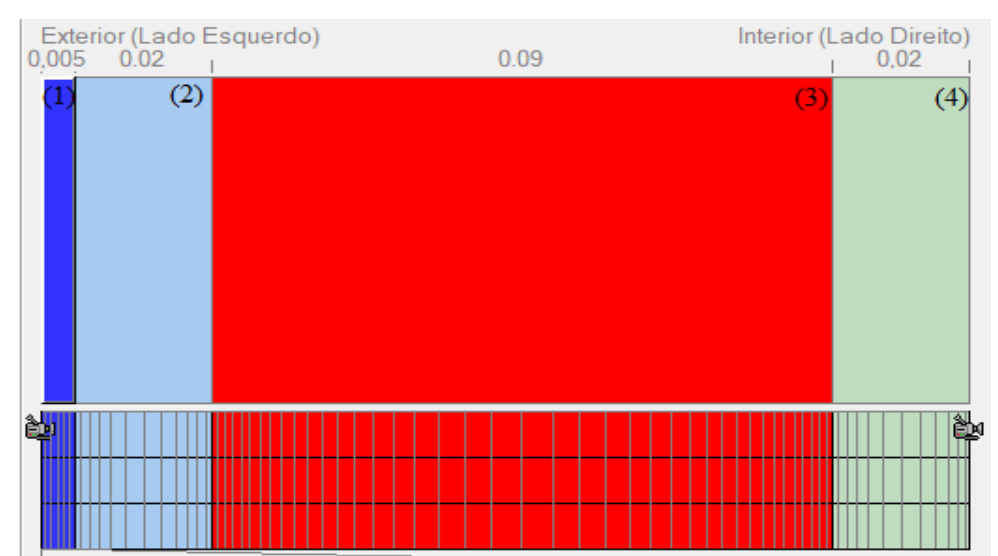

Figura 1. Sistema construtivo avaliado: (1) placa cerâmica; (2) argamassa externa/emboço; (3) bloco cerâmico; (4) argamassa externa.

Além disso, as propriedades dos materiais componentes das camadas do sistema estudado foram obtidas previamente de ensaios, realiados por outros pesquisadores, de massa específica aparente, porosidade, permeabilidade ao vapor de água para o cálculo do Fator de resistência à difusão de vapor de água; coeficiente de absorção de água ou coeficiente de capilaridade; curva higroscópica. Esse ensaios e seus resultados são descritos no Relatório Interno do LEM-UnB (BAUER; NASCIMENTO; CASTRO, 2015).

\subsection{Ações dos agentes associados à temperatura.}

\subsubsection{Radiação total, temperatura superficial máxima e mínima e amplitude térmica máxima.}

A partir dos dados de temperaturas superficiais obtidos foi possível determinar as temperaturas máximas e mínimas assim como as amplitudes térmicas diárias (diferença diária entre temperatura máxima e mínima na fachada) ao longo do período de simulação, calculadas como mostrado em (1). Com os dados de radiação solar determinou-se a radiação solar acumulada na fachada ao longo do período de simulação, somando os valores diários observados em cada fachada.

$$
\Delta T=t_{\text {máx }}-t_{\text {mín }}
$$

Onde $\Delta \boldsymbol{T}$ é a amplitude térmica, $\boldsymbol{t}_{\boldsymbol{m} \text { á }}$ é a temperatura máxima diária da superfície da fachada e $\boldsymbol{t}_{\boldsymbol{m} \mathbf{n}}$ é a temperatura mínima diária da superfície da fachada.

\subsubsection{Choque térmico.}

No presente artigo, como parte do estudo dos efeitos associados à temperatura, foi analisado o choque térmico (Zanoni, 2015). Para tanto, foi determinada a frequência de ocorrência dos eventos denominados como choque térmico pleno e choque térmico atenuado para um ano de simulação em todas as fachadas. O primeiro configura-se quando há um diferença de temperatura superficial superior a $8{ }^{\circ} \mathrm{C}$ no intervalo de 1 hora, já o segundo quando a mesma diferença de temperatura é verificada em um intervalo de $2 \mathrm{~h}$. A equações para choque térmico pleno e choque térmico atenuado são mostrados em (2) e (3), respectivamente.

$$
\Delta T_{c h}=t_{n}-t_{n-1}
$$




$$
\Delta T_{c h}=t_{n}-t_{n-2}
$$

\subsection{3 Índice de intensidade da temperatura.}

$\mathrm{Na}$ intenção de analisar os efeitos associados á temperatura, calcula-se para todas as fachadas o índice de intensidade da temperatura (Iit), o qual leva em consideração os efeitos dos ciclos e valores alcançados de temperatura superficial nos sistemas (Nascimento, 2016). Este índice é estabelecido com base nas variações de temperaturas e suas frequências de ocorrência em um determinado intervalo pré-estabelecido e é uma análise da amplitude térmica obtendo-se uma medida ponderada em relação a frequência. A Tabela 3 mostra os quatro intervalos iguais nos quais são distribuídas as faixas de variação da amplitude térmica consideradas na metodologia de Nascimento (2016), as quais consideram intervalos inferiores a $11,5^{\circ} \mathrm{C}$ (faixa 1) até intervalos superiores a $27,7^{\circ} \mathrm{C}$ (faixa 4). Por fim, o Iit é calculado como é mostrado em (4).

Tabela 3. Faixas de intervalo de amplitude térmica consideradas para o cálculo de Iit.

\begin{tabular}{|c|c|c|}
\hline Faixa & $\Delta \mathrm{T}\left({ }^{\circ} \mathrm{C}\right)$ & $\Delta \mathrm{T}$ médio $\left({ }^{\circ} \mathrm{C}\right)$ \\
\hline 4 & $>27.7$ & 31.8 \\
\hline 3 & 19.7 a 27.7 & 23.7 \\
\hline 2 & 11.5 a 19.6 & 15.6 \\
\hline 1 & $<11.5$ & 7.5 \\
\hline
\end{tabular}

$I_{i t}=\sum \frac{\Delta T_{\text {médio }} \times f_{n}}{f_{\text {total }}}$

Onde $\boldsymbol{I}_{i t}$ é o índice de intensidade da temperatura $\left({ }^{\circ} \mathrm{C}\right), \Delta \boldsymbol{T}_{\boldsymbol{m}}$ édio é a amplitude de temperatura média da faixa de ocorrência $\left({ }^{\circ} \mathrm{C}\right), \boldsymbol{f}_{\boldsymbol{n}}$ é a frequência de ocorrência da faixa "n" de $\Delta \mathrm{T}$ e $\boldsymbol{f}_{\text {total }}$ é a frequência de todas às ocorrências das quatro faixas de estudo no ano, ou seja, $\boldsymbol{f}_{\text {total }}=\mathbf{3 6 5}$.

\subsubsection{Chuva dirigida.}

O estudo de Chuva dirigida é realizado pelo método de quantificação do WUFI, no qual são obtidos os dados horários com um total de 8760 horas para precipitação, velocidade e direção do vento de modo a alimentar as simulações computacionais (Zanoni, 2015). A partir disso a intensidade de chuva dirigida é calculada em (5).

$R_{w d r}=R_{2} \cdot R_{h} \cdot V \cdot \cos (D-\theta)$ 
Onde $\boldsymbol{R}_{\boldsymbol{w} \boldsymbol{d} \boldsymbol{r}}$ é a Intensidade de chuva dirigida $(\mathrm{mm} / \mathrm{h}) ; \boldsymbol{R}_{\boldsymbol{h}}$ é a Precipitação em superfície horizontal (mm); $\boldsymbol{R}_{\mathbf{2}}$ é o Coeficiente dependente da localização na fachada $(\mathrm{s} / \mathrm{m})$; $\boldsymbol{V}$ é a Média horária da velocidade do vento a $10 \mathrm{~m}$ de altura $(\mathrm{m} / \mathrm{s}) ; \boldsymbol{D}$ é a Direção do vento (ângulo a partir do Norte); $\boldsymbol{\theta}$ Orientação da fachada: ângulo entre o Norte e a direção normal à parede. $\mathrm{O}$ valor de $\boldsymbol{R}_{\mathbf{2}}$ depende da altura do edifício e da localização da área de estudo, nesse caso o valor adotado é 0,2 para edifícios altos, parte superior maior que 20 metros. Como dados de saída da simulação foram fornecidas os valores de precipitação incidente nas fachadas. Desse modo, foi possível calcular o valor acumulado anual em $1 / \mathrm{m}^{2}$ de chuva dirigida para as diferentes zonas bioclimáticas.

\section{RESULTADOS}

\subsection{Radiação total, temperatura superficial máxima e amplitude térmica máxima.}

Os resultados de radiação total incidente e das amplitudes térmicas superficiais máximas absolutas, calculadas como mostrado em (1), das fachadas Norte das oito zonas bioclimáticas brasileiras são mostrados na Figura 2. Nota-se que os maiores valores de amplitude térmica ocorrem para a zona $\mathrm{Z6}\left(38,5^{\circ} \mathrm{C}\right)$ seguidos de $\mathrm{Z} 2\left(37,5^{\circ} \mathrm{C}\right)$ e $\mathrm{Z} 1\left(37,15^{\circ} \mathrm{C}\right)$, já o menor valor é verificado na $\mathrm{Z8}\left(22,64^{\circ} \mathrm{C}\right)$ e as demais zonas apresentam valores intermediários. No que se refere à incidência de radiação solar, a Z4 apresenta maior valor $\left(1.014 .409 \mathrm{~W} / \mathrm{m}^{2}\right)$ seguida da Z6 $\left(995.347 \mathrm{~W} / \mathrm{m}^{2}\right)$, enquanto isso $\mathrm{Z8}$ apresenta o menor valor de incidência de radiação (743.527 $\mathrm{W} / \mathrm{m}^{2}$ ). Ademais, observa-se que a incidência de radiação solar está associada ao ganho de temperatura em cada fachada principalmente no que se nota nas zonas Z8 e Z6, com exceção a zona Z4 a qual apresentou maior incidência de radiação e a terceira menor amplitude térmica.

No caso da incidência de radiação total, as zonas Z4 e Z6 seriam críticas e Z8 a que apresenta condições de exposição mais amenas. No entanto, a temperatura superficial e a amplitude térmica na superfície, por serem resultados da interação entre a radiação solar e a elevação da temperatura do ar ao longo do dia, são melhores indicadores das variações higrotérmicas do que o total da irradiação solar incidente (Zanoni, 2015). Nesse caso, Z6 e Z7 seriam zonas críticas, por apresentarem maior amplitude térmica (Figura 2) e maior temperatura superficial (Figura 3) ao longo do ano, respectivamente.

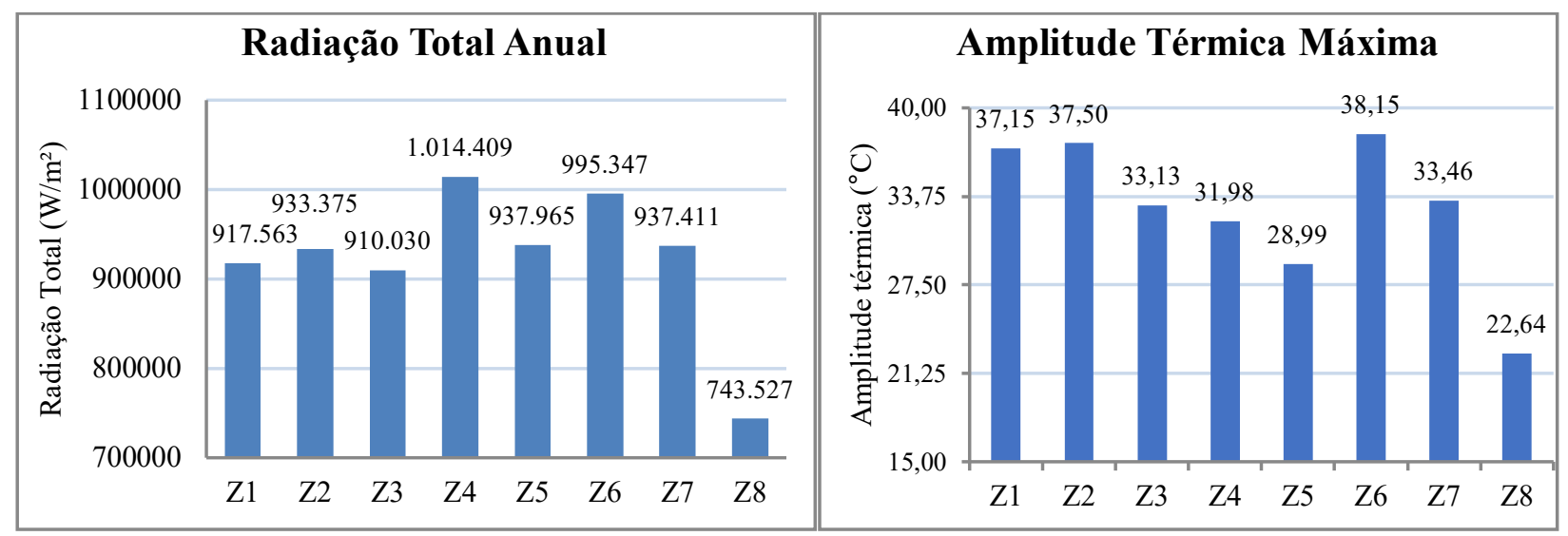

Figura 2. Radiação total acumulada e Amplitude térmica máxima em um ano de simulação.

Os resultados de temperatura máxima e mínima absoluta anual para cada uma das fachadas são mostradas na Figura 3. Nota-se que as temperaturas mínimas, as quais ocorrem quando não há incidência de radiação sobre a fachada, apresentam grandes distinções entre si. Z2, Z1 e Z3 
apresentam os menores valores de temperatura $2,99^{\circ} \mathrm{C}, 5,48^{\circ} \mathrm{C}$ e $6,98^{\circ} \mathrm{C}$, respectivamente. Enquanto isso, $\mathrm{Z8}$ apresenta temperatura mínima mais elevada $\left(22,07^{\circ} \mathrm{C}\right)$ e menor valor de temperatura máxima $\left(46,16^{\circ} \mathrm{C}\right)$ o que condiz com amplitudes térmicas reduzidas, como mostrado na Figura 2.
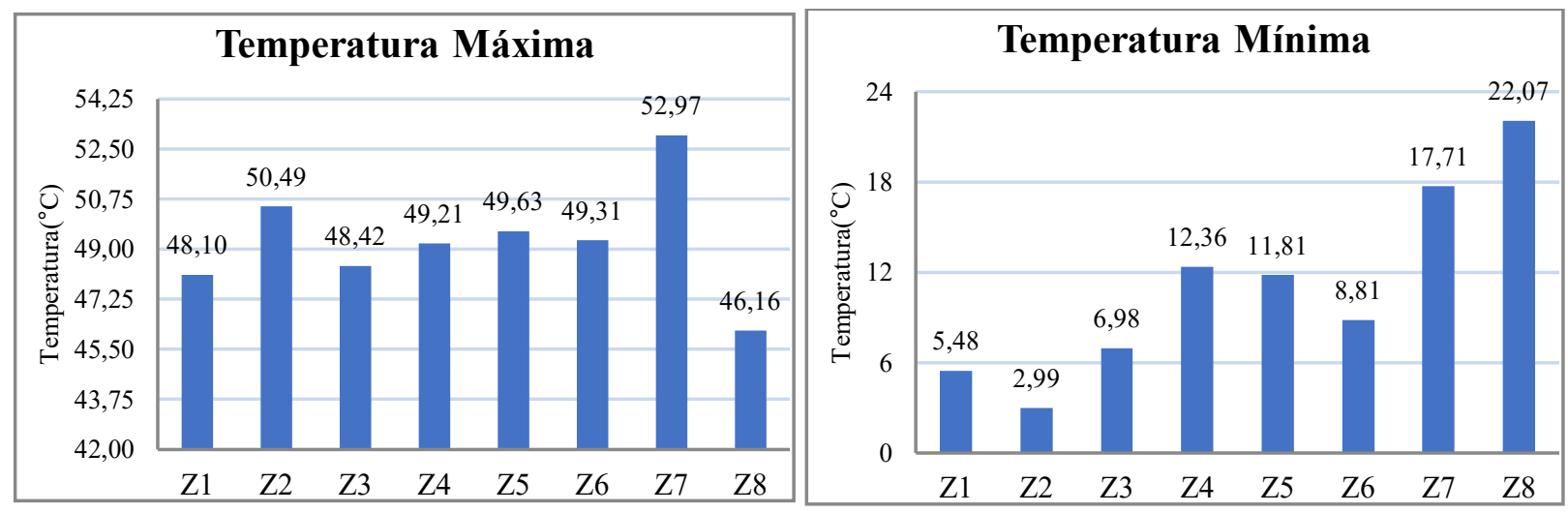

Figura 3. Temperatura superficial máxima e mínima absolutas em um ano de simulação para cada zona bioclimática.

\subsection{Choque térmico.}

Com relação aos resultados de choque térmicos, são apresentadas na Figura 4 as frequências de ocorrência de choques plenos para todas as fachadas. Ressalta-se a maior frequência na zona Z6, na qual $1,59 \%$ das medidas se caracterizam como choques térmicos plenos, seguido de $Z 1$ $(0,92 \%)$ e Z4 (0,79\%). As zonas Z5 e Z7 apresentaram as menores frequências $0,16 \%$ e $0,19 \%$, respectivamente.

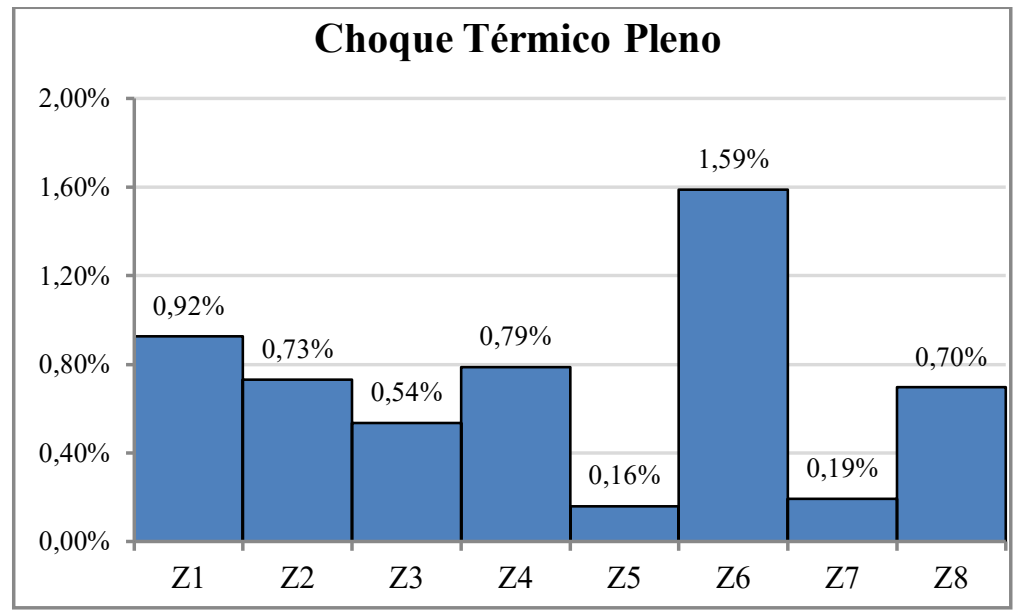

Figura 4. Frequência de ocorrência de choque térmico pleno para um ano de simulação.

Os picos de choques plenos estão concentrados para todas as zonas nos meses de maio a julho como mostrado na Tabela 4, na qual é exposta a distribuição mensal do número de choques para cada zona. Ao longo de um ano, é possível verificar em que mês ocorre o maior número de choques térmicos para cada zona isolada. Dessa forma, Z1 e Z5 tem seus respectivos picos de ocorrência de choques térmicos plenos em julho, Z3, Z4, Z6 e Z7 em junho e Z2 e Z8 em maio. Nas zonas de 1 a 7 os choques térmicos diminuem nos meses de outubro a março chegando a zero em um ou mais meses desse período. No entanto isso não ocorre na Z8 que apresenta os eventos de choques térmicos melhor distribuídos ao longo do ano. 
Tabela 4. Número de eventos de choque térmico pleno mensal ao longo de um ano de simulação.

\begin{tabular}{|c|c|c|c|c|c|c|c|c|c|c|c|c|c|}
\hline \multicolumn{10}{|c|}{ Choque Térmico Pleno } \\
\hline & jan & fev & mar & abr & mai & jun & jul & ago & set & out & nov & dez & soma \\
\hline Z1 & 1 & 1 & 1 & 9 & 14 & 18 & $\mathbf{2 5}$ & 9 & 1 & 1 & 0 & 1 & 81 \\
\hline Z2 & 0 & 1 & 0 & 4 & $\mathbf{2 1}$ & 10 & 7 & 12 & 7 & 0 & 0 & 2 & 64 \\
\hline Z3 & 1 & 0 & 0 & 1 & 6 & $\mathbf{2 5}$ & 14 & 0 & 0 & 0 & 0 & 0 & 47 \\
\hline Z4 & 1 & 1 & 0 & 4 & 17 & $\mathbf{2 3}$ & 19 & 0 & 0 & 1 & 0 & 3 & 69 \\
\hline Z5 & 0 & 0 & 0 & 0 & 1 & 5 & $\mathbf{6}$ & 1 & 1 & 0 & 0 & 0 & 14 \\
\hline Z6 & 4 & 1 & 3 & 10 & 13 & $\mathbf{5 3}$ & 38 & 12 & 0 & 0 & 1 & 4 & 139 \\
\hline Z7 & 0 & 1 & 3 & 3 & 2 & $\mathbf{6}$ & 2 & 0 & 0 & 0 & 0 & 0 & 17 \\
\hline Z8 & 5 & 5 & 6 & 7 & $\mathbf{1 0}$ & 5 & 2 & 3 & 1 & 4 & 5 & 8 & 61 \\
\hline
\end{tabular}

A Figura 5 mostra a frequência de ocorrência de choques atenuados. Novamente a Z6 apresenta maior ocorrência com $13,65 \%$ de medidas caracterizadas como choques atenuados, seguida da Z4 com 10,88\%. Z8, Z5 e Z2 apresentam os menores valores de frequência sendo estes 2,84\%, $3,35 \%$ e 4,95\%, respectivamente. Nota-se que na Z6 ocorrem aproximadamente 5 vezes mais choques atenuados em comparação a Z8. Z4 e Z1 também apresentam valores elevados de frequência (10,88\% e 10,31\%, respectivamente) seguidos de Z3 e Z7 (8,75\% e 8,60\%).

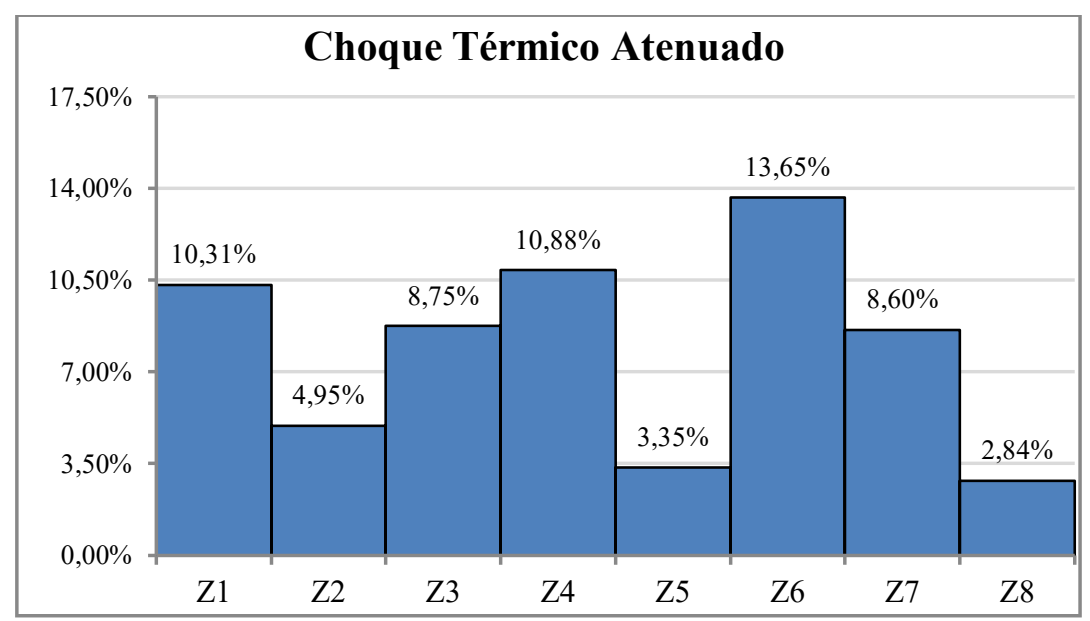

Figura 5. Frequência de ocorrência de choque térmico atenuado para um ano de simulação. 
Quando a distribuição desses eventos ao longo do ano nota-se que eles também ocorrem nos meses centrais do ano, nesse caso de maio a agosto, como mostra a Tabela 5. Apenas a Z5 apresenta meses sem a ocorrência de choques térmicos, os quais são os meses de novembro e dezembro. Z2 e Z8 tiveram seu pico de ocorrência no mês de maio, Z4 e Z6 em junho, Z1, Z5 e Z7 em julho e Z3 em agosto. É possível perceber uma melhor distribuição de ocorrências ao longo do ano em Z8, onde o número de ocorrência varia de 12 em setembro a 32 em maio, já em outras zonas há uma variação mais elevada entre os meses de menor e maior ocorrência como na Z6 que apresenta menor ocorrência em novembro com 14 eventos e maior ocorrência em junho com 211 eventos.

Tabela 5. Número de eventos de choque térmico atenuado mensal ao longo de um ano de simulação.

\begin{tabular}{|l|l|l|l|l|l|l|l|l|l|l|l|l|l|}
\hline \multicolumn{10}{|c|}{ Choque térmico Atenuado } \\
\hline & jan & fev & mar & abr & mai & jun & jul & ago & set & out & nov & dez & soma \\
\hline Z1 & 13 & 24 & 64 & 112 & 146 & 103 & $\mathbf{1 9 0}$ & 114 & 78 & 46 & 4 & 9 & 903 \\
\hline Z2 & 6 & 14 & 39 & 65 & $\mathbf{8 5}$ & 38 & 50 & 62 & 44 & 27 & 1 & 3 & 434 \\
\hline Z3 & 6 & 25 & 72 & 106 & 127 & 115 & 76 & $\mathbf{1 2 8}$ & 73 & 28 & 5 & 5 & 766 \\
\hline Z4 & 6 & 18 & 52 & 120 & 148 & $\mathbf{1 8 2}$ & 177 & 143 & 71 & 16 & 10 & 10 & 953 \\
\hline Z5 & 2 & 1 & 5 & 32 & 46 & 56 & $\mathbf{8 2}$ & 44 & 22 & 3 & 0 & 0 & 293 \\
\hline Z6 & 27 & 33 & 76 & 121 & 175 & $\mathbf{2 1 1}$ & 207 & 170 & 84 & 56 & 14 & 22 & 1196 \\
\hline Z7 & 10 & 11 & 32 & 80 & 155 & 145 & $\mathbf{1 5 7}$ & 114 & 40 & 3 & 2 & 4 & 753 \\
\hline Z8 & 18 & 15 & 22 & 29 & $\mathbf{3 2}$ & 20 & 29 & 19 & 12 & 18 & 14 & 21 & 249 \\
\hline
\end{tabular}

As zonas com maior frequência de choque térmico apresentam condições de exposição críticas e aquelas que apresentam menores frequências condições mais amenas. Destaca-se a Zona Z6 que apresentou maior frequência tanto para choques térmicos plenos quanto para atenuados como situação mais crítica. Por fim, a zona Z5 apresentou frequências reduzidas nos dois tipos de choques considerados e a Z8 menor frequência para choques atenuados.

\section{3. Índice de intensidade da temperatura.}

A Figura 6 mostra os resultados de Iit para todas as fachadas correspondentes a cada zona bioclimática. Os resultados levam em consideração valores de variação de temperatura para um ano de simulação. Assim como notado nos resultados de amplitude térmica máxima e choque térmico, a zona Z6 apresentou valores mais críticos $(22,55)$ quanto à ação de agentes de temperatura. Da mesma forma, a zona Z8 e Z5 apresentaram os menores valores $(16,53$ e 16,55) 
e Iit. Z2, Z4 e Z7 apresentaram valores também elevados se comparados aos demais e Z3 e Z1 apresentaram valores intermediários.

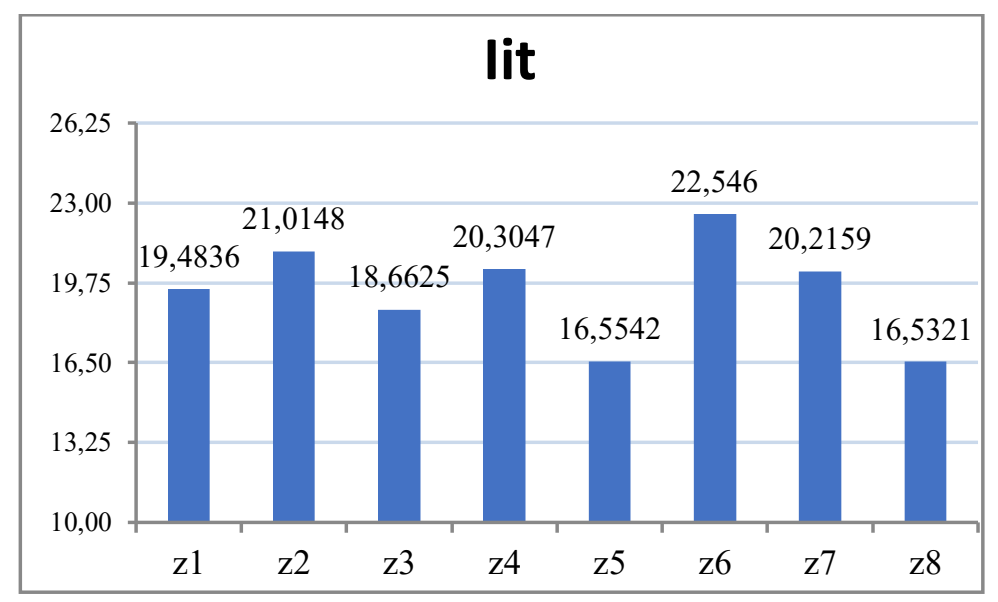

Figura 6. Valores de Iit para todas as zonas bioclimáticas.

\subsection{Chuva dirigida.}

A chuva dirigida foi analisada apenas em termos de incidência precipitação total anual $\left(1 / \mathrm{m}^{2}\right)$, Figura 7, para as mesmas fachadas de orientação Norte, de forma completar a análise dos agentes de degradação realizada. Nota-se que para a zona Z8 é a mais crítica para esse caso $\left(1 / \mathrm{m}^{2}\right)$ apresentando valor cerca de 6 vezes mais elevado que a zona Z5 de menor incidência $\left(18,21 / \mathrm{m}^{2}\right)$, contrastando com os resultados anteriores. As zonas Z4 $\left(100,121 / \mathrm{m}^{2}\right)$ e Z3 $\left(71,721 / \mathrm{m}^{2}\right)$ também apresentam valores elevados em comparação às demais. Por fim, Z1 (52,19 1/ $\left.\mathrm{m}^{2}\right)$ e Z6 $(59,67$ $\left.1 / \mathrm{m}^{2}\right)$ apresentam valores intermediários enquanto Z2 $\left(35,57 \mathrm{l} / \mathrm{m}^{2}\right)$ e $\mathrm{Z} 7\left(30,57 \mathrm{l} / \mathrm{m}^{2}\right)$ valores mais amenos de incidência de chuva.

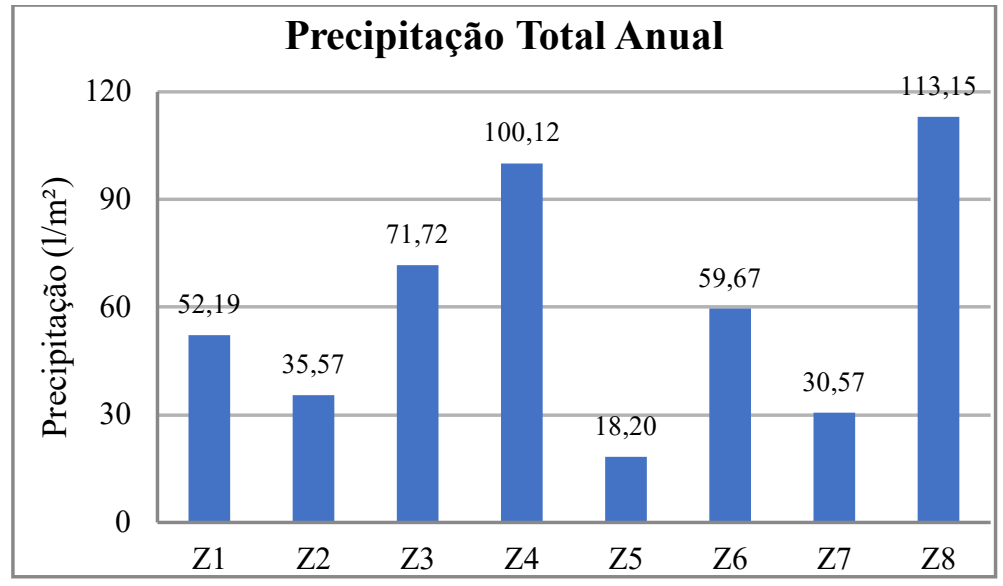

Figura 7. Valores de chuva dirigida acumulada para um não de simulação em todas as zonas bioclimática

\section{CONCLUSÃO}

Propôs-se a investigar a ação dos agentes de degradação associados à temperatura e a chuva dirigida nas fachadas de edifícios localizados em diferentes zonas bioclimáticas brasileiras. Nesse contexto, é possível identificar zonas que apresentam condições críticas de exposição aos agentes de degradação como forma de contribuir para o conhecimento das diferentes condições que 
desencadeiam o processo de degradação no Brasil. Dessa forma, foi possível levantar os seguintes comportamentos:

-A zona bioclimática 6, representada pela cidade de Goiânia, proporciona condições mais críticas de exposição em relação a amplitude térmica, choque térmico atenuado e pleno e índice de intensidade da temperatura;

-A zona bioclimática 8, representada pela cidade de Belém, apesar de apresentar condição de exposição mais amena para os agentes associados a temperatura (radiação solar, amplitude térmica, choque térmico atenuado e Índice de intensidade da temperatura) se mostra crítica para exposição á chuva dirigida.

- As zonas Z1, Z2, Z3, Z5 e Z7, representados pela cidade de Curitiba, Santa Maria, Florianópolis, Niterói e Picos, respectivamente, apresentaram valores de incidência de radiação total anual na fachada Norte semelhantes. No entanto Z1 e Z2 apresentam elevadas amplitudes térmicas se comparada às demais.

- Em relação aos choques térmicos, Z1 e Z4, representadas pelas cidades de Curitiba e Brasília, apresentaram valores elevados de frequência e Z5 as menores frequências. Em relação ao Iit, o maior valor foi da fachada Norte da Zona Z6, sendo que as das zonas Z2, Z4 e Z7 também apresentaram valores elevados. Em contrapartida as zonas Z5 e Z8 apresentaram as condições mais amenas.

- Em relação à Chuva dirigida, as fachadas Norte das zonas Z8 e Z4 apresentaram mais exposição considerando valores anuais de precipitação e a Zona Z5 apresentou a condição mais amena de exposição.

A partir disso conclui-se que as fachadas Norte analisadas nas 8 zonas bioclimáticas brasileiras possuem diferentes condições de exposição aos agentes associados à temperatura e à chuva dirigida. Além disso, como contribuição o estudo fornece dois rankings relativos à exposição aos agentes investigados. Primeiramente com relação aos agentes relacionados à temperatura têm-se, em ordem decrescente, as zonas mais expostas: Z6-Z2-Z4-Z7-Z1-Z3-Z5-Z8. Por fim em relação à chuva dirigida têm-se, também em ordem decrescente, as zonas mais expostas: Z8-Z4-Z3-Z6Z1-Z2-Z7-Z5.

\section{REFERENCIAS}

Associação Brasileira de Normas Técnicas. (2005). NBR 15220-3: Desempenho térmico de edificações Parte 3: Zoneamento bioclimático brasileiro e diretrizes construtivas para habitações unifamiliares de interesse social. Rio de Janeiro.

Barbosa, A. S.(2013) "Estudo Numérico-Computacional e Analítico do Choque Térmico em Fachadas de Edificações”. Dissertação (Mestrado). Masters Thesis, Programa de Pós-Graduação em Estruturas e Construção Civil, Universidade de Brasília, Brasília-DF.

Bauer, E.; Nascimento, M.L.M.; Castro, E.K.(2015). "Parâmetros e ensaios físicos de materiais e componentes da fachada. Relatório interno” - Laboratório de Ensaio de Materiais (LEM) $\mathrm{UnB} / \mathrm{ENC}$.

Bezerra, L. M.; Uchôa, J. C., Araújo, J. A.; Bonilla, J. (2018) Experimental and Numerical Investigation of Fatigue in Base-Rendering Mortar Used in Façades Undergoing Thermal Cycles. Journal of Materials in Civil Engineering. v. 30. n. 8. pp.1-14.

https://doi-org.ez54.periodicos.capes.gov.br/10.1061/(ASCE)MT.1943-5533.0002319 
Dornelles, K. A.; Roriz, M. (2007). “A ilusão das cores na identificação da absortância solar de superficies opacas”. In: IX ENCAC E VII ELACAC, 2007, Ouro Preto. Anais. Ouro Preto: ANTAC.

Freitas, A. S. S. L. A. (2011), "Avaliação do comportamento hidrotérmico de revestimentos exteriores de fachadas devido à acção da chuva incidente", Masters Thesis. Faculdade de Engenharia da Universidade do Porto. Porto, Portugal. 170 p.

Gaspar, P., Brito, J. Mapping Defect Sensitivity in External Mortar Renders. Journal of Construction and Building Materials, v. 19(8), p. 571-578, 2005.

https://doi.org/10.1016/j.conbuildmat.2005.01.014

Moscoso, Y.F.M. (2013), "Estudo numérico e experimental de tensões atuantes na argamassa colante de fachadas de edificações sob ação da fadiga termo-mecânica”. Masters Thesis, Universidade de Brasília, Brasília. p. 142.

Nascimento, M. (2016), “ Aplicação da simulação higrotérmica na investigação da degradação de fachadas de edifícios”, Masters Thesis, Universidade de Brasília, Brasília, 2016.p. 127

Roriz, M. (2012). Correções nas Irradiâncias e Iluminâncias dos arquivos EPW da Base ANTAC. Grupo de Trabalho sobre Conforto e Eficiência Energética de Edificações. ANTAC Associação Nacional de Tecnologia do Ambiente Construído. São Carlos - SP.

Souza, J.(2019), "Impacto dos fatores de degradação sobre a vida útil de fachadas de edificios", doctoral thesis, Universidade de Brasília, Brasília, Brasil, p. 101.

Saraiva, A. G. (1998), “Contribuição ao Estudo de Tensões de Natureza Térmica em Sistemas de Revestimento Cerâmico de Fachada”. Masters Thesis. Universidade de Brasília. Brasília, Brasil. $164 \mathrm{p}$.

Zanoni, V.(2015), “ Influência dos agentes climáticos de degradação no comportamento higrotérmico de fachadas em Brasília”. Doctorial Thesis, Universidade de Brasília, Brasília, 2015. p. 253. 